\title{
Experimental determination of heat partition in elastohydrodynamic contacts.
}

\author{
Amjad Al-Hamood ${ }^{\mathrm{a}, \mathrm{b}}$, A Clarke ${ }^{\mathrm{a}}, \mathrm{H}_{\mathrm{P}}$ Evans $^{\mathrm{a}}$ \\ a School of Engineering, Cardiff University, United Kingdom. \\ b College of Engineering, Karbala University, Karbala, Iraq.
}

\section{ABSTRACT}

Experimental and theoretical analyses are reported to study the heat generation and partition in an elastohydrodynamic rolling/sliding point contact. Heat is generated within the lubricant in the Hertzian region by shearing and compression of the oil film. This heat is essentially conducted to the contacting surfaces as the amount convected from the Hertzian zone by the lubricant can be neglected due to the very low lubricant mass flowrate.

A two-disk test rig was used for the experimental tests using crowned, superfinished $76.2 \mathrm{~mm}$ diameter disks fixed on parallel shafts. Each disk was fitted with six thermocouples in two rows of three located $3 \mathrm{~mm}$ and $6 \mathrm{~mm}$ below the surface to measure the temperature distribution of the disks during the tests. In addition, the disks were insulated on both plane sides by ceramic washers to minimise heat transfer to the surroundings over those surfaces.

A numerical model was developed to calculate the circumferential mean disk temperature distribution in the outer $6 \mathrm{~mm}$ annular ring using the inner row of thermocouples to provide a boundary condition. The model was used to predict the temperature distribution for given values of the fraction of the total heat entering the fast disk, $\beta$, and the heat transfer coefficient, $h$, for the disk running surfaces. Minimisation of error between predicted and experimentally measured temperatures at the thermocouple positions, together with consideration of the physical relationship between fast and slow shaft heat transfer coefficients led to the conclusion that $\beta$ lies in the range 0.71 to 0.77 for the experiments reported in the paper and that approximately $75 \%$ of the frictional heat dissipated within the lubricant film flows 
into the faster disk. This result indicates that the likely mechanism of heat dissipation within the lubricant film is by slip at or near the faster surface and that consideration of heat partition is a more discerning judge of lubricant rheological behaviour than the usual consideration of traction measurements alone.

Keywords: Elastohydrodynamic lubrication; thermal; heat partition

\section{INTRODUCTION}

The earliest tribological studies of frictional heating in sliding were carried out for the dry contact situation by Blok [1], Jaeger [2] and Archard [3], for example. These studies were based on consideration of the surface temperature rise under a moving source of heat. Blok considered the case of a semiinfinite body subjected to a concentrated moving heat source, and determined the maximum surface temperature rise at both low and high Peclet numbers. These studies introduced the concept of flash temperature - the transient temperature rise associated with the passage of the surface through the contact.

Dry contact simulations have been extended to include consideration of asperity interactions within the nominal contact area with contributions by Barber [4], Vick and Furey [5], Salti and Laraqi [6] and Gao et.al. [7] being representative examples.

Surface heating in a lubricated rolling/sliding contact is complicated by the need to consider the lubricant as well as the solid bodies. Olver [8] considered the estimation of bulk and flash temperatures in various designs of disk and shaft geometries in disk machines used for lubricant testing. Heat partition was calculated by taking the average contact temperatures to be equal and taking convective cooling from the disk and shaft surfaces into account.

The majority of the solutions outlined above deal with the division of frictional heat between the surfaces in contact by assuming that either the maximum or average temperatures on the surfaces are equal or, in the numerical solutions, by matching temperatures on the surfaces at all points in contact. 
This is a plausible way to proceed for dry contacts, but the presence of an oil film separating the surfaces in lubricated contacts makes this assumption questionable, as recognised by Olver [8].

Fluid film lubrication of heavily loaded concentrated contacts is achieved by means of the elastohydrodynamic lubrication (EHL) mechanism where the pressures developed in the lubricant lead to a significant increase in viscosity together with elastic deflection of the surfaces. When sliding of the surfaces occurs, heat is generated within the film by shearing and compressive heating of the lubricant. The dominant heat transfer mechanism is that of conduction perpendicular to the film. If heat is generated throughout the thickness of the film, the highest temperatures are developed within the film itself, and the temperature gradient necessary to transport the heat by conduction to the surfaces ensures that the surfaces are at lower and generally different temperatures. Thus, the lubricant film must be considered in any study of thermal behaviour of lubricated contacts.

An early analytical analysis which considered the oil film and removed the restriction of equal surface temperatures was published by Merritt [9]. His work was based on that of Blok, but he included the partition of heat between the two surfaces as a variable, which could be adjusted to vary the surface temperatures of the two surfaces. He then used this theory to analyse the results of some disk-machine experiments. He found that more of the heat (around 60\%) flowed into the faster disk. In addition, both measured temperatures and his calculations showed that the faster disk achieved a higher surface temperature than the slower disk. Merritt went on to consider conceptually the heat generation and temperature gradients within the oil film, and found that he could not reconcile the measured and calculated heat partition and surface temperatures with his assumption that the rate of heat generation was uniform across the film thickness. He concluded that there must exist some asymmetry in the mechanism of heat generation within the oil film.

Clarke et al. [10] used numerical heat conduction models to calculate the bulk and total contact temperatures in disk machine experiments carried out by Patching et al. [11]. Temperature measurements 
for the disks obtained from single thermocouples located 3mm below the running track were used together with a numerical model of each shaft and disk to infer the heat partition. The authors reached the conclusion that the majority of the heat dissipated at the contact did indeed pass into the faster disk, with values of between $62 \%$ and $75 \%$ obtained for a large range of experimental conditions with both axially ground and superfinished disks. However these results required heat transfer coefficients to be specified for all surfaces of the rotating disk and shaft, a source of some uncertainty. Subsequent work by Clarke et al. [12] considered the question of heat partition behaviour as a means of discriminating between various candidate rheological and viscosity models in smooth surface EHL simulations. It was found that, when compared to the experimental results of [10], the mechanism for heat dissipation within the contact which gave similar heat partition results to Clarke's thermal model was one which involved slip at or near the faster surface.

The current paper reports findings from a study carried out using detailed measurements of the temperature distributions in the disks in order to eliminate uncertainties arising from the heat transfer coefficient values assumed in the previous analysis. 


\section{NOTATION}

A B E G

$b$

c

$d$

D

F

$h$

$h_{f}$

$h_{s}$

$H_{c}$

$k$

$k_{c}$

$k_{0}$

$n$

$q$

$r$

$r_{1}$

$r_{2}$

$t$

$T$

$T_{0}$

$\tilde{T}$

$u_{f}$

$u_{s}$

$U_{s}$

w

$\mathrm{Z}$

$\alpha$

$\beta$

$\varepsilon$

$\chi$

$\Lambda$

$\rho$

$v$

$\xi$
Hertzian minor semi-dimension

$\mathrm{m}$

Constants in equations (8) and (9)

$\mathrm{m}$

$\mathrm{m}$

$\mathrm{J} / \mathrm{kgK}$

Lubricant specific heat

Thickness of ceramic washers

$\mathrm{m}$

$\mathrm{m}$

$\mathrm{N}$

$\mathrm{W} / \mathrm{m}^{2} \mathrm{~K}$

$\mathrm{W} / \mathrm{m}^{2} \mathrm{~K}$

$\mathrm{W} / \mathrm{m}^{2} \mathrm{~K}$

m

$\mathrm{W} / \mathrm{mK}$

$\mathrm{W} / \mathrm{mK}$

$\mathrm{W} / \mathrm{mK}$

$\mathrm{W} / \mathrm{m}^{2}$

$\mathrm{m}$

$\mathrm{m}$

m

$\mathrm{S}$

${ }^{\circ} \mathrm{C}$

${ }^{\circ} \mathrm{C}$

${ }^{\circ} \mathrm{C}$

$\mathrm{m} / \mathrm{s}$

$\mathrm{m} / \mathrm{s}$

$\mathrm{m} / \mathrm{s}$

$\mathrm{m}$

$\mathrm{m}$

$\mathrm{m}^{2} / \mathrm{s}$

Thermal diffusivity

Heat partition factor

Error measure in calculated temperatures

${ }^{\circ} \mathrm{C}$

Parameter $\chi=\left(u_{f} / u_{s}\right)^{n}$

Specific film thickness

Lubricant density

Kinematic viscosity of fluid

$\mathrm{kg} / \mathrm{m}^{3}$

$\mathrm{m}^{2} / \mathrm{s}$

Slide/roll ratio 


\section{EXPERIMENTAL RIG}

The two-disk test rig used for the experimental tests has parallel shafts fitted with $76.2 \mathrm{~mm}$ diameter disks. The disks are $9.5 \mathrm{~mm}$ wide and are crowned with a $304.8 \mathrm{~mm}$ radius in the axial direction so as to prevent edge contact. They are pressed onto the shafts using a heavy interference fit, ensuring good thermal contact between the disk and shaft, and are located axially against the shaft shoulder and retained by locking rings.

The shafts that support the disks are gear connected so that the rig operates in a power recirculating mode. Toothed belt stages are included in the drive train to provide flexibility in selecting the shaft speed ratio. The disks rotate in opposite directions and constitute a rolling/sliding elliptical point contact with aspect ratio $b / a=4$. The disk surface velocities are in the same direction relative to the contact and are parallel to the minor axis of the contact ellipse with the slide/roll ratio fixed by the selected shaft speed ratio. The slow shaft bearings are located in a pivoted housing yoke so that load can be applied to the contact between the rotating disks by loading the housing with a hydraulic ram. The disks used in the experiments were super-finished to an $R a$ value (arithmetic mean of the absolute values of the roughness profile ordinates) of $0.04 \mu \mathrm{m}$ so that surface roughness was not significant as the contacts operated with specific film thickness values in the range $\Lambda=2$ to 7 .

Each disk is sandwiched between a pair of $6 \mathrm{~mm}$ thick ceramic washers which are fitted to the plane faces to provide thermal insulation so that very little heat transfer to the surroundings can take place via the disk washer interfaces. The washers had an outside diameter of $76 \mathrm{~mm}$ and were slightly tapered to ensure that they would not contact each other when fitted to the test disks under load. Figure 1 is a photograph of the test head where the two disks with their fitted ceramic washers can be seen in a central position. The slow shaft can be seen towards the top of the figure with its bearings adjacent to the disk located in the tilting housing yoke. The fast shaft is towards the bottom of the figure with its bearings mounted in two fixed housings. Splined gear couplings at the top left of the test head connect the test 
shafts to the gear connected driving shafts. The test disks are enclosed in a thin walled two part steel enclosure during the test which includes oil feed pipes that direct temperature controlled lubricant sprays to the inlet and the outlet of the contact. These pipes and the upper half of the enclosure are not shown in the figure for clarity.

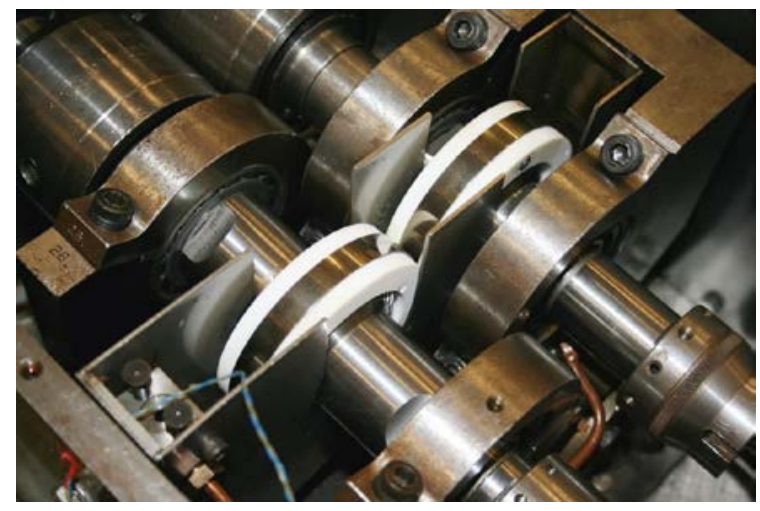

Figure 1. Photograph of test disks fitted to shafts in the test rig.

Each disk was fitted with six thermocouples in two rows of three, located $3 \mathrm{~mm}$ and $6 \mathrm{~mm}$ below the surface, to measure the temperature distribution of the disks during the tests. The thermocouples were located at the bottom of blind holes drilled through the plane sides of the disk and potted with a hightemperature thermally conductive cement. The thermocouples were located at different circumferential positions and the wires were fed through radial holes in the hollow thick walled shafts to a pair of silver/graphite slip rings at the shaft ends. The thermocouples measure the mean temperature during the rotation of the shaft at their respective radial and axial positions. Figure 2 shows a section of a shaft centred on the disk with the positions of the six thermocouples indicated. In each case the thermocouple hole is drilled from the closest plane face of the disk. The crown applied to the disk ensures that the contact is central on the running surfaces. 


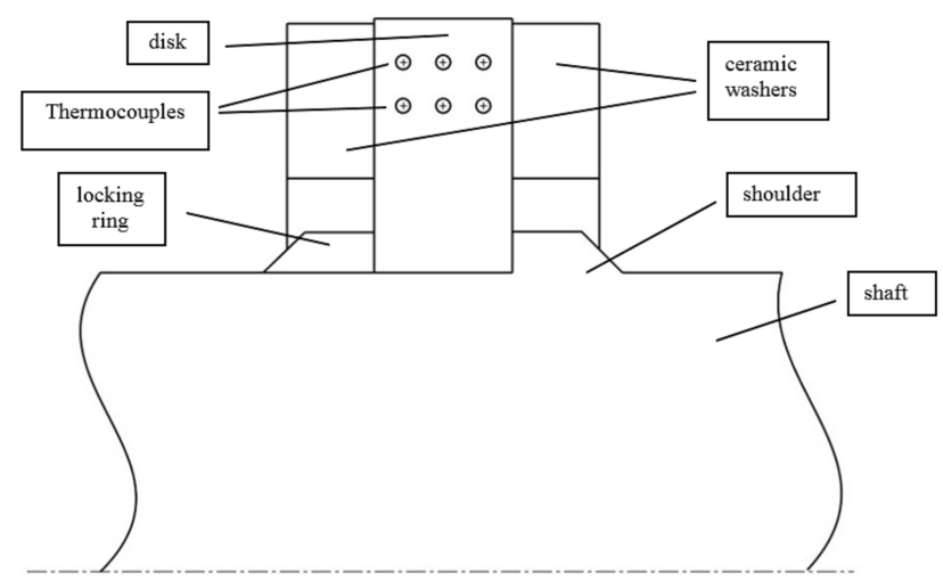

Figure 2. Section of the fast shaft

Each thermocouple was connected to individually isolated signal conditioning hardware so as to avoid the spurious readings that occur otherwise due to ground loops between thermocouples. In addition the thermocouples, once installed in the test disks, were calibrated against a reference platinum-resistance thermometer in an environmental chamber, in order to improve measurement accuracy to better than $\pm 0.2^{\circ} \mathrm{C}$. During tests the applied load and friction force were recorded together with the twelve thermocouple temperatures. These measurements were taken at $0.1 \mathrm{~s}$ intervals and an example of the measured thermocouple temperature at point a (as defined in Figure 4) is shown in Figure 3. 


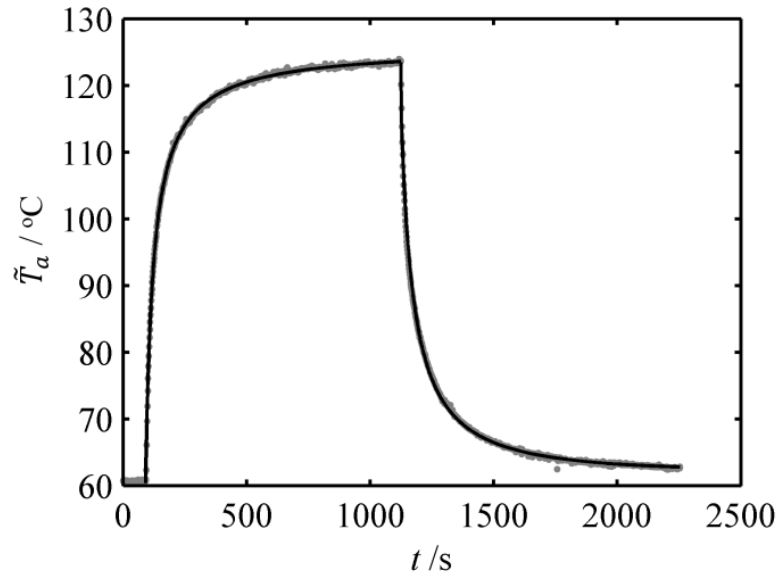

Figure $\quad 3$. Transient temperature at example thermocouple

As the thermocouple recorded temperatures represent average values over several revolutions, any rapid variations (other than at onset and end of loading) are caused by electrical noise. To avoid this influencing subsequent calculations the experimental temperature data (shown as points) were fitted with separate smooth curves over the loaded and unloaded periods as shown in Figure 3.

\section{THERMAL MODEL OF TEST DISK}

In order to relate the measured disk temperatures to the amount of heat entering the disks a heat partition parameter, $\beta$, is introduced. This is defined as the fraction of the total heat dissipated at the contact that enters the fast disk. The fraction of the dissipated heat entering the slow disk is then $1-\beta$ as the amount of heat convected out of the contact by the lubricant film separating the surfaces is orders of magnitude smaller than that conducted into the disks. This can be easily verified as the rate at which heat is convected out of the Hertzian contact area by motion of the oil in the lubricant film can be approximated by the expression 


$$
Q_{\text {film }}=b H_{c}\left(u_{f}+u_{s}\right) \rho c \Delta T
$$

where $\Delta T$ is the mean temperature rise of the lubricant in passing through the Hertzian contact area, while the rate at which heat is dissipated at the contact is

$$
Q_{f r}=F\left(u_{f}-u_{s}\right)
$$

Evaluating $Q_{f i l m}$ and $Q_{f r}$ for the contacts used in the experiment using data for film temperature and thickness from [11] and [12] shows that $Q_{f r}$ exceeds $Q_{\text {film }}$ by a factor of between 500 and 1200, and consequently that $Q_{\text {film }}$ can be disregarded as being negligible.

A numerical model of the heat flow in the outer annular ring of the disk was then developed to calculate the mean temperature during rotation as a function of time. Figure 4 shows the annular ring considered, JLMN, which has inner radius $r_{2}$ and outer radius $r_{1}$, which is the disk surface. The positions of the thermocouples are indicated and labelled a, b, c, d, e and f.

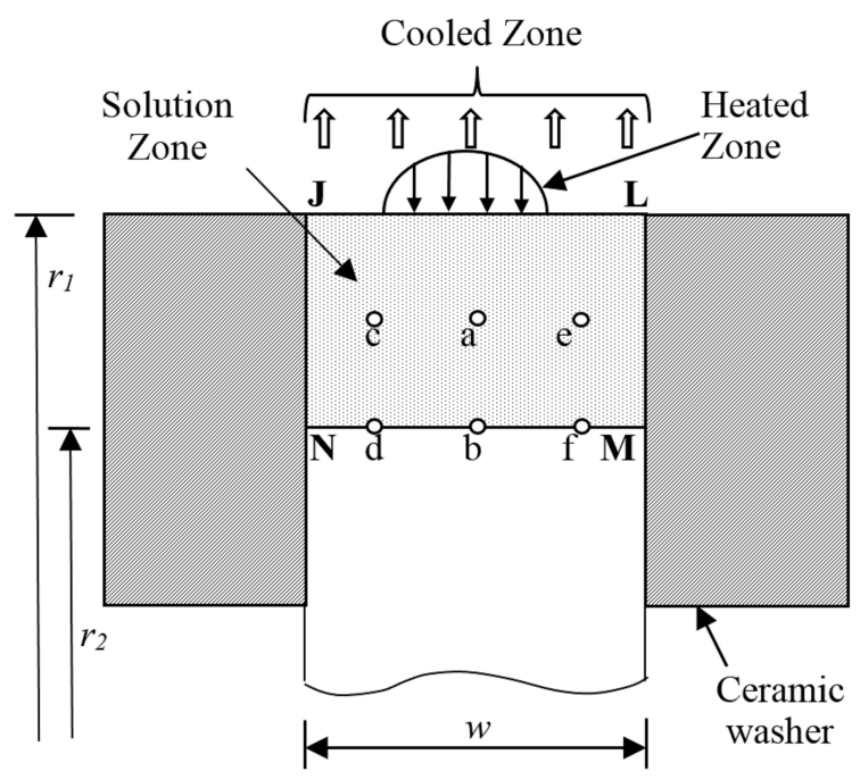

Figure 4 Section of disk showing solution zone JLNM with thermocouple positions a to $\mathrm{f}$. 
The variation of temperature, $T(r, z, t)$, in the annular ring is given by the equation

$$
\frac{1}{\alpha} \frac{\partial T}{\partial t}=\frac{\partial^{2} T}{\partial r^{2}}+\frac{1}{r} \frac{\partial T}{\partial r}+\frac{\partial^{2} T}{\partial z^{2}}
$$

Here, $T$ is the mean circumferential temperature and consequently there are no terms involving derivatives with respect to the circumferential coordinate, $\theta$.

Equation (3) is discretised using central finite differences over a rectangular mesh of points covering area JLMN. The problem is formulated using the Crank-Nicolson implicit method with the solution for each timestep obtained by Gauss Seidel relaxation.

Radius $r_{2}$ is the position of the inner ring of thermocouples and those measured values are used to provide a temperature boundary condition $T\left(r_{2}, z, t\right)$ for all times. $T\left(r_{2}, z, t\right)$ is obtained by fitting a parabola or other suitable curve to the three measured temperatures $T_{d}(t), T_{b}(t)$, and $T_{f}(t)$. (Note that measured temperatures $T_{a}(t)$ to $T_{f}(t)$ are fitted with individual smooth curve fits as discussed in section 4.)

The thermal conductivity of the ceramic washer is significantly smaller than the steel disk, and the axial boundary condition on surfaces JN and LM reflect this by linking the interface heat flux in the disk to that in the ceramic washer according to

$$
k \frac{\partial T}{\partial z}= \pm k_{c} \frac{T_{i}-T_{0}}{d}
$$

where $T_{0}$ is the ambient temperature and $T_{i}$ is the interface temperature, and the sign differs for the two sides (negative for the face whose outward normal is the positive $z$ direction).

The boundary condition imposed on surface JL, which is the running surface of the disk, reflects the heating caused within the contact and cooling to the surroundings outside the contact area. The heat input is averaged over the surface and applied to the contact track using a semi elliptic distribution to reflect the pressure distribution. The surface cooling is specified in terms of a heat transfer coefficient so that on boundary JL 


$$
k \frac{\partial T}{\partial r}=q-h\left(T_{s}-T_{0}\right)
$$

where $q$ is the heat flux due to heating at the contact, $h$ is the heat transfer coefficient and $T_{s}$ is the surface temperature. Outside the contact region (i.e. where $z^{2}>b^{2}$ ) the heat flux $q$ is set to zero. Inside the contact region, for the fast disk

$$
q(z, t)=\frac{1.5 \beta F(t)\left(u_{f}-u_{s}\right)}{4 \pi r_{2} b}\left(1-\frac{z^{2}}{b^{2}}\right)
$$

and for the slow disk $\beta$ is replaced by $1-\beta$ in equation (6).

The initial temperature distribution at the start of the transient analysis is interpolated from the six thermocouple measurements at the time that the load is applied to the disks, and the analysis continues for the duration of the test time.

\section{ANALYSIS METHOD FOR DETERMINING HEAT PARTITION BASED ON EXPERIMENTAL RESULTS}

The tests were analysed by carrying out a series of analyses for each disk with systematic variation of the specified parameters $\left(\beta, h_{f}\right)$ and $\left(\beta, h_{s}\right)$ for the fast and slow disks respectively. Each of these analyses results in a different temperature distribution within the disk at the end of the heating period when the thermocouple signals become essentially steady. A measure of the discrepancy between the calculated and observed temperature fields was introduced as

$$
\varepsilon=\frac{\left|T_{c}-\tilde{T}_{c}\right|+2\left|T_{a}-\widetilde{T}_{a}\right|+\left|T_{e}-\widetilde{T}_{e}\right|}{4}
$$

where $\widetilde{T}_{c}$ is the model temperature at point $\mathrm{C}$, etc. The value of $\varepsilon$ in ${ }^{\circ} \mathrm{C}$ obtained at each $(\beta, h)$ point is plotted in the form of a contour plot as illustrated in figures 5a and $5 b$ for the fast and slow disks for one 
of the experiments. These $\varepsilon$ values are obtained be averaging over the last $200 \mathrm{~s}$ of the heating period where the measurements indicate that the disks have attained near steady state conditions. The example used in this section is a test at a maximum Hertzian pressure of $1.4 \mathrm{GPa}$, sliding speed of $20 \mathrm{~m} / \mathrm{s}$ and entrainment speed of $16.3 \mathrm{~m} / \mathrm{s}$.

(a)

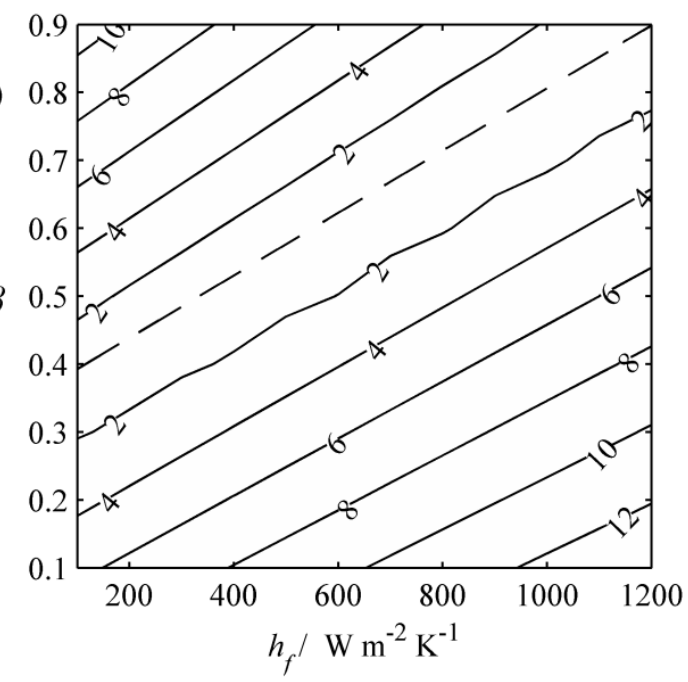

(b)

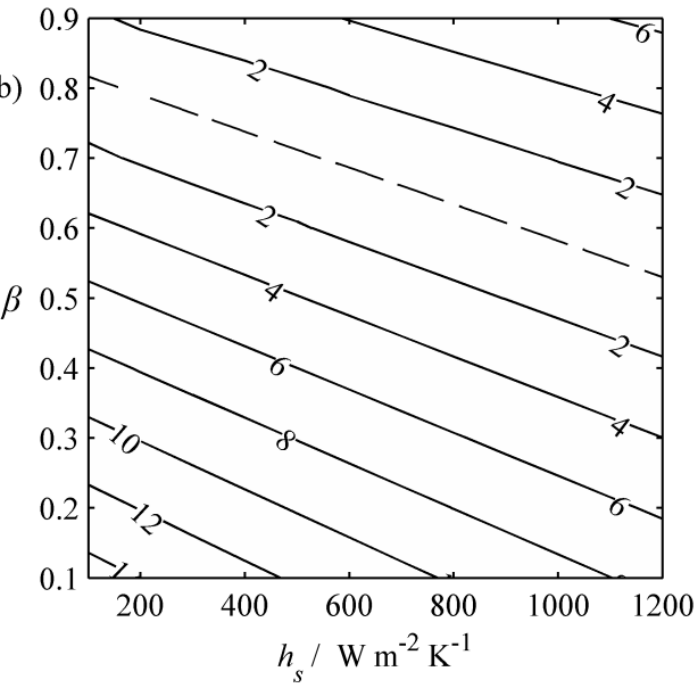

Figure 5. Contours of $\varepsilon$ (in ${ }^{\circ} \mathrm{C}$ ) obtained for the (a) fast disk and (b) slow disk, respectively.

When plotted in the $\left(\beta, h_{f}\right)$ and $\left(\beta, h_{s}\right)$ parameter space for the two disks both of these contour plots show similar behaviour. The error contours are broadly parallel in each figure and it is clear that the minimum value of $\varepsilon$ occurs on the dashed line that is located between the two parallel contours with the lower $\varepsilon$ value. It is not possible to identify a clear minimum $\varepsilon$ position from these results but there are clear linear relationships between the parameters that correspond to the lowest values of $\varepsilon$. These are the equations of the dashed lines in Figure 5 and can be expressed as

$$
\beta=A+B h_{f}
$$




$$
\beta=E+G h_{s}
$$

It should be noted that the values of the coefficients A, B, E and G are different for each experiment being modelled. The smallest value of $h_{f}$ and $h_{s}$ that was used in these plots is $100 \mathrm{~W} / \mathrm{m}^{2} \mathrm{~K}$ which gives rise to extreme values of $\beta=0.53$ and $\beta=0.82$ from equations (8) and (9). The calculated temperature distributions for these values of $\beta$ are shown in Figures 6 and 7. Figure 6 shows the calculated temperature distributions for the two disks using the value $\beta=0.53$ with the values of $h_{f}$ and $h_{s}$ corresponding to the minimum error obtained from equations (8) and (9). Figure 7 shows the corresponding results for $\beta=0.82$. The results show that the surface temperatures in the fast disk are highest when the high $\beta$ value is adopted whereas the slow disk surface temperatures are lowest at the high $\beta$ value. The fast disk surface temperature varies from $173{ }^{\circ} \mathrm{C}$ for $\beta=0.53$ to $179{ }^{\circ} \mathrm{C}$ for $\beta=0.82$, and the slow disk surface temperature varies from $124{ }^{\circ} \mathrm{C}$ for $\beta=0.53$ to $119{ }^{\circ} \mathrm{C}$ for $\beta=0.82$. Using intermediate values of $\beta$ shows that this variation of maximum temperature is systematic as $\beta$ is varied, as might be expected. 

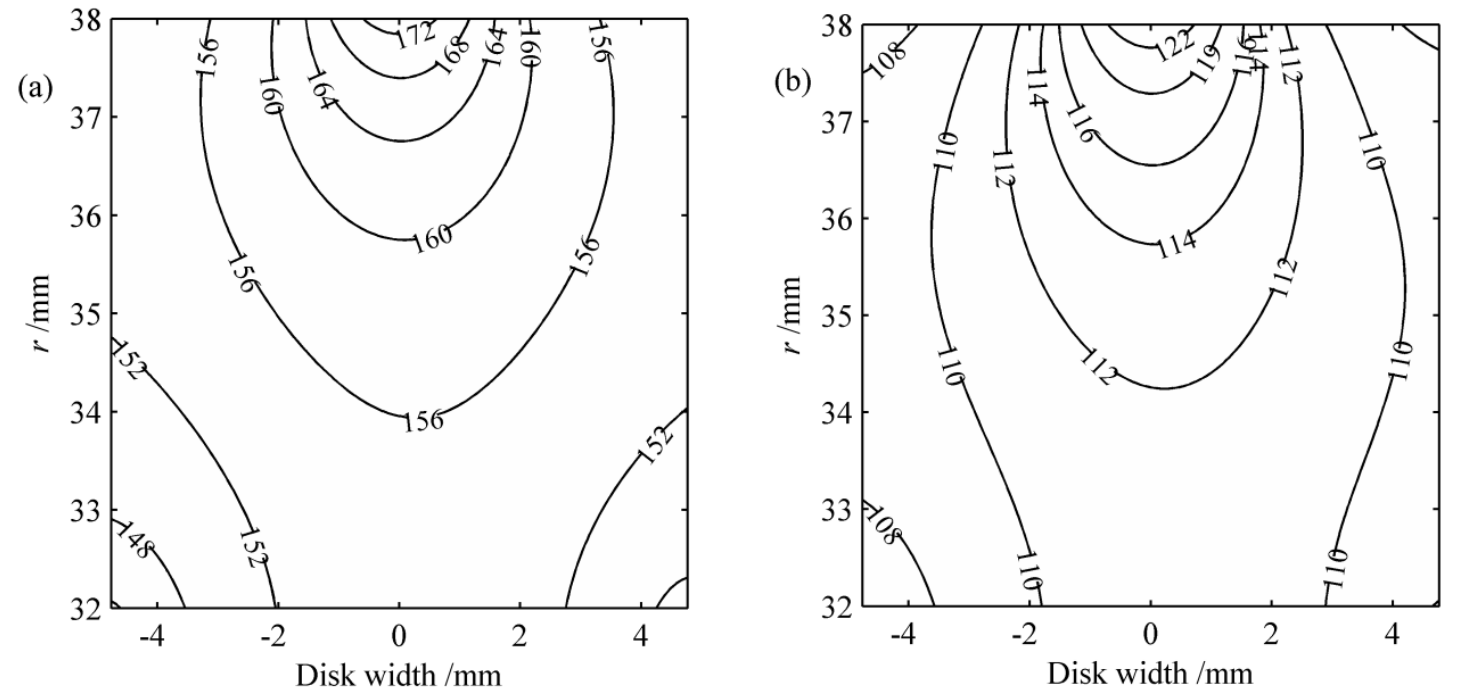

Figure 6. Calculated temperature distributions in ${ }^{\circ} \mathrm{C}$ for (a) fast disk and (b) slow disk with $\beta=0.53$.
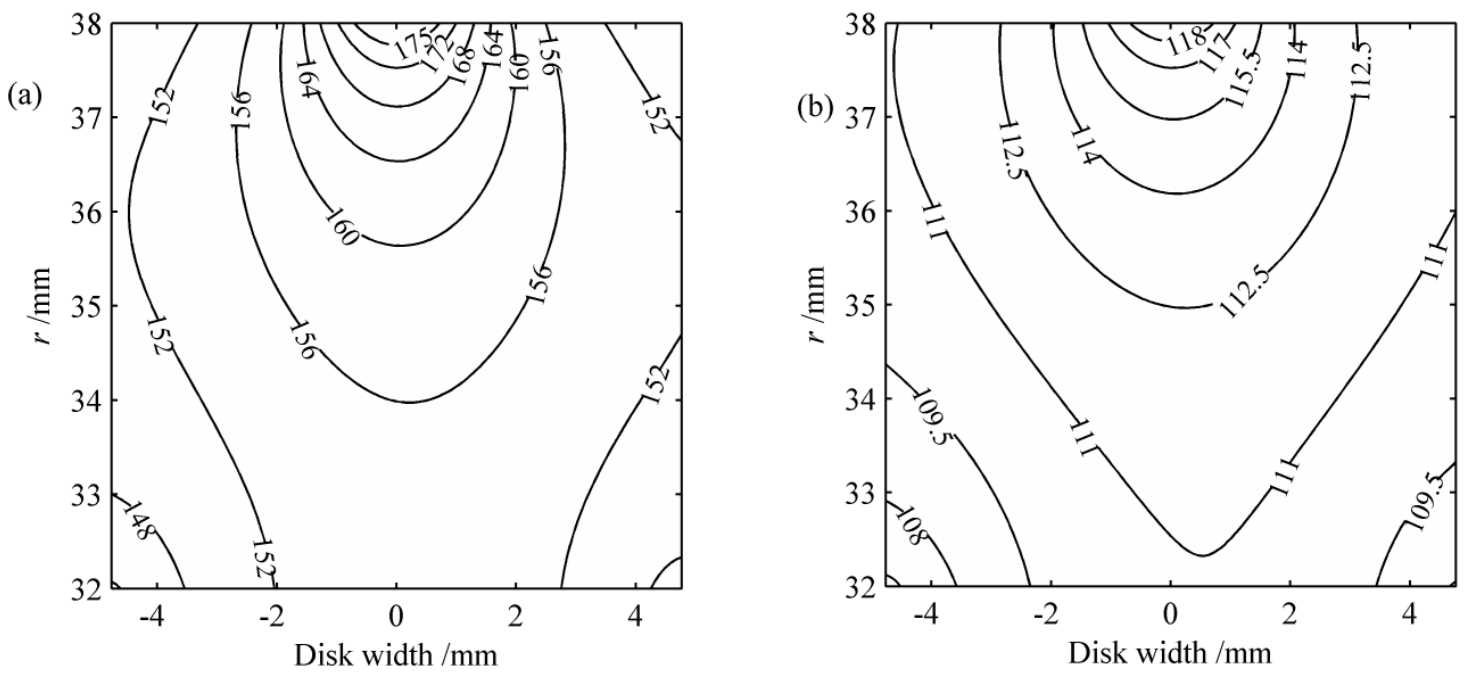

Figure 7. Calculated temperature distributions in ${ }^{\circ} \mathrm{C}$ for

(a) fast disk and (b) slow disk with $\beta=0.82$. 
Merritt [9] approached the difficulty in determining the heat transfer coefficient by using the cooling part of the temperature curve in an approximate way. However this approach is subject to the difficulty that the potted thermocouples have a time constant that is significant in terms of the initial rapidity of the measured decline in temperature when the load is removed. The current authors are examining the option of characterising the transient response so that the initial temperature decline can be used to determine the $h_{s}$ and $h_{f}$ values accurately, but have not yet succeeded to overcome the difficulties associated with this approach.

It is possible, however, to identify the value of $\beta$ by introducing the relationship that can be expected to exist between $h_{f}$ and $h_{s}$. Several studies have been carried out to evaluate the heat transfer from a cylinder rotating about its horizontal axis as it is one of the important heat transfer problems in engineering. Anderson and Saunders [13], Dropkin and Carmi [14], Cardone et al. [15], and Ozerdem [16], carried out experimental programmes to study convection heat transfer of a horizontal cylinder rotating in a fluid. In these experimental studies the Nusselt number, Nu, and the Reynolds number, Re, were found to follow the relation

$$
\mathrm{Nu}=C(\mathrm{Re})^{n}
$$

where $\mathrm{C}$ is constant which in the current notation becomes

$$
\frac{h D}{k_{0}}=C\left(\frac{u D}{v}\right)^{n}
$$

The heat transfer coefficients then become

$$
h_{f}=C \frac{k_{0}}{D}\left(\frac{D}{v}\right)^{n}\left(u_{f}\right)^{n} \text { and } h_{s}=C \frac{k_{0}}{D}\left(\frac{D}{v}\right)^{n}\left(u_{s}\right)^{n}
$$

so that the ratio of the heat transfer coefficients can be expected to be 


$$
h_{f} / h_{s}=\left(u_{f} / u_{s}\right)^{n}=\chi
$$

Solving equations (8) (9) and (12) leads to the result

$$
\beta=\frac{\chi B E-A G}{\chi B-G}
$$

Values of power $n$ obtained in the literature vary between 0.57 and 0.8 , and the generally accepted value is 0.667 , which is the value used for the calculations in the current paper.

For the case illustrated in figures (5), (6) and (7) equation (13) then gives $\beta=0.75$ and figure 8 shows the temperature distributions in the two disks for this value of $\beta$. It can be seen that the maximum surface temperatures obtained from the model are $177^{\circ} \mathrm{C}$ for the fast disk and $120{ }^{\circ} \mathrm{C}$ for the slow disk, and consideration of the sequence of temperature distributions presented in Figures (6) (8) and (7) confirm the systematic temperature changes for both disks as $\beta$ is increased.
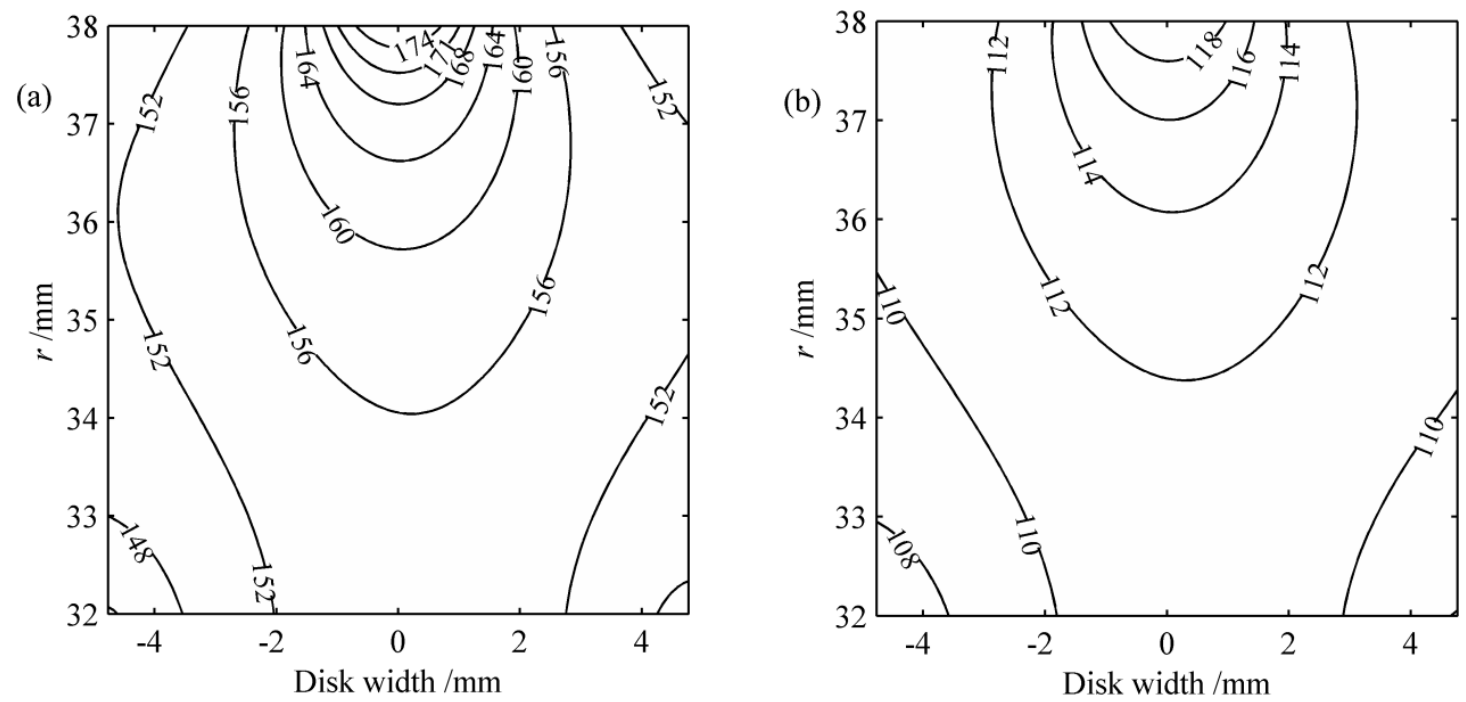

Figure 8. Calculated temperature distributions in ${ }^{\circ} \mathrm{C}$ for (a) fast disk and (b) slow disk with $\beta=0.75$. 


\section{RESULTS}

Experiments were run at four sliding speeds : 10,13, 16 and $20 \mathrm{~m} / \mathrm{s}$ with the gearing set to give a slide/roll ratio of 1.24 so that $u_{f} / u_{s}=4.26$. At each of these sliding speeds, tests were carried out with loads of 850, 1460, 1850 and $2320 \mathrm{~N}$ corresponding to maximum Hertzian contact pressures of 1.0, 1.2, 1.3 and $1.4 \mathrm{GPa}$, respectively. The lubricant used was Mobil-Jet 2 with an oil supply temperature of $60{ }^{\circ} \mathrm{C}$. Previous scuffing tests by Patching et.al. [11] using the same experimental rig established that fast disk temperatures $\left(T_{a}\right)$ exceeded $180{ }^{\circ} \mathrm{C}$ when scuffing occurred in all tests and the test conditions adopted were chosen so as to ensure that scuffing would not occur.

The experimental measurements were processed as described in sections 5 and 6 to deduce the experimental value of $\beta$ for each test, and these are given in Table 1. The disk temperature distribution calculated for these $\beta$ values were examined and the mean surface temperatures over the Hertzian heated zone, $T_{H f}$ and $T_{H s}$, were determined and are also given in Table 1.

Table 1. Experimental values obtained for $\beta$.

\begin{tabular}{|c|c|c|c|c|c|}
\hline $\begin{array}{c}\bar{u} \\
/ \mathrm{ms}^{-1}\end{array}$ & $\begin{array}{c}U_{s} \\
/ \mathrm{ms}^{-1}\end{array}$ & $\begin{array}{c}p_{H Z} \\
/ \mathrm{GPa}\end{array}$ & $\beta$ & $\begin{array}{c}T_{H f} \\
/{ }^{\circ} \mathrm{C}\end{array}$ & $\begin{array}{c}T_{H s} \\
/{ }^{\circ} \mathrm{C}\end{array}$ \\
\hline 8.06 & 10.0 & 1.0 & 0.71 & 82.5 & 67.6 \\
\hline 8.06 & 10.0 & 1.2 & 0.75 & 106.9 & 79.4 \\
\hline 8.06 & 10.0 & 1.3 & 0.73 & 116.6 & 84.3 \\
\hline 8.06 & 10.0 & 1.4 & 0.73 & 132.5 & 92.9 \\
\hline 10.5 & 13.0 & 1.0 & 0.75 & 89.3 & 72.1 \\
\hline 10.5 & 13.0 & 1.2 & 0.76 & 116.3 & 84.9 \\
\hline 10.5 & 13.0 & 1.3 & 0.75 & 129.6 & 91.5 \\
\hline 10.5 & 13.0 & 1.4 & 0.74 & 145.2 & 100.3 \\
\hline 12.9 & 16.0 & 1.0 & 0.77 & 93.9 & 75.7 \\
\hline 12.9 & 16.0 & 1.2 & 0.76 & 122.6 & 90.1 \\
\hline 12.9 & 16.0 & 1.3 & 0.77 & 139.1 & 97.0 \\
\hline 12.9 & 16.0 & 1.4 & 0.76 & 157.8 & 107.5 \\
\hline 16.3 & 20.0 & 1.0 & 0.72 & 95.1 & 78.3 \\
\hline 16.3 & 20.0 & 1.2 & 0.75 & 126.7 & 94.7 \\
\hline 16.3 & 20.0 & 1.3 & 0.76 & 149.5 & 105.3 \\
\hline 16.3 & 20.0 & 1.4 & 0.75 & 169.9 & 116.9 \\
\hline
\end{tabular}


The values of $\beta$ obtained from the experiments are all in the range of 0.71 to 0.77 for the conditions considered indicating that around three-quarters of the heat dissipated passes into the higher speed disk. In each case the faster disk has a higher surface temperature than the slower disk This almost constant value of $\beta$ is generally consistent with calculations previously presented by the authors in [10] for the same conditions which were based on single temperature measurements in each disk, and a simpler thermal analysis of both shafts gave values of $\beta$ in the range 0.63 to 0.74 with a mean value of 0.68. For the analysis in [10] nominal values for heat transfer coefficients on the plane and cylindrical surfaces of the rotating shafts and disks were taken from the literature. In the current model the analysis is confined to the outer annular ring of the disks and there is only one heat transfer coefficient that influences the calculated disk temperature as per equation (5). The only assumption made is that, for a given test, the heat transfer coefficients for the fast and slow disks are related by equation 12 with an assumed value of $n=0.667$. To test the sensitivity of the calculated $\beta$ values to the value on $n$ adopted the results for two of the test conditions were repeated for a range of $n$ values between 0.5 and 0.8 . The variation in the resulting $\beta$ values is presented in figure 9 which shows that the value of $\beta$ obtained is relatively insensitive to the value of $n$ assumed for the calculation. 


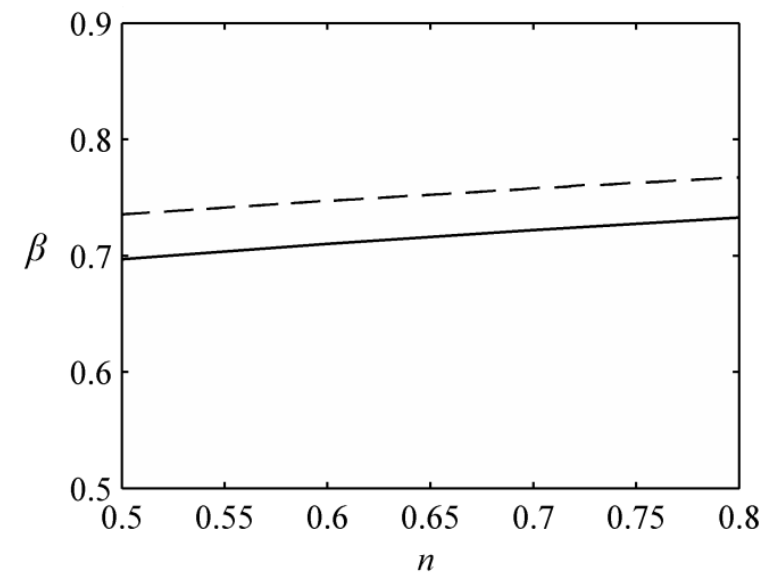

Figure 9. Variation of $\beta$ with assumed value of $n$ : solid line $U_{s}=10 \mathrm{~m} / \mathrm{s}$, $p_{\mathrm{Hz}}=1 \mathrm{GPa}$; broken line $U_{\mathrm{s}}=20 \mathrm{~m} / \mathrm{s}, p_{\mathrm{Hz}}=1.4 \mathrm{GPa}$, 


\section{DISCUSSION AND CONCLUSIONS}

The heat partition values determined from the experiments reported in this paper indicate that in the rolling sliding contacts considered $75 \%$ of the dissipated heat passes into the faster moving surface. This is consistent with previous results presented by the authors. The work presented by Merritt [9] indicates that if "the oil film offers no resistance to heat flow" and the flash contact temperatures of the disks are equal, then calculation of equal Block flash temperatures for the disks leads to

$$
\beta=\frac{\sqrt{u_{f}}}{\sqrt{u_{f}}+\sqrt{u_{s}}},
$$

In addition, if $\beta$ exceeds this value, the flash temperature of the faster disk will exceed that of the slower disk. For the disk speed ratio used in the experiments this equation gives a value of $\beta=0.67$. The values of $\beta$ given in Table 1 are higher than this as might be expected given that the mean surface temperature difference between the disks varies between $15{ }^{\circ} \mathrm{C}$ and $53{ }^{\circ} \mathrm{C}$ over the experiments, with the higher temperature in the faster disk. Numerical analyses of the EHL oil film using a variety of viscosity and non-Newtonian rheological models [12] have shown that the values of $\beta$ obtained from thermal EHL considerations are generally less than 0.5 if the shear heating is dissipated in a distributed way across the oil film, and values of $\beta$ greater than 0.6 are only obtained if the rheological model results in lubricant slip within the film at or near to the higher temperature surface. It is instructive to note that, for all of the combinations of viscosity and rheological models considered in [12], the calculated frictional traction matched that measured in experiment. This suggests that the heat partition behaviour of a contact is a far more discerning discriminator between lubricant models than matching of traction force. The technique is also of use in explaining the temperature results of, for example, Snidle et al. [17] who observed the thermal effects of DLC coatings acting as a barrier to heat flow in disk machine tests where a DLC coated disc was run against a non-coated counterface. 
The results obtained in this study suggest that for the lubricant considered (Mobil Jet 2):

- Approximately $75 \%$ of the heat dissipated in the rolling sliding contact passes into the faster moving surface which attains a higher bulk temperature than that of the slower moving surface.

- This thermal behaviour is indicative of lubricant slip at the faster moving surface.

\section{ACKNOWLEDGEMENTS}

Amjad Al-Hamood is grateful to the Ministry of Higher Education and Scientific Research, Iraq, and to the College of Engineering, University of Karbala, Karbala, Iraq, for support of his studies at Cardiff University. This research received no specific grant from any funding agency in the public, commercial, or not-for-profit sectors

\section{REFERENCES}

1. Blok H. Theoretical study of temperature rises at surfaces of actual contact under oiliness conditions. Proceedings of the IMechE - General discussion on lubrication 1937; 2: 222-235.

2. Jaeger JC. Moving sources of heat and the temperature of sliding contacts. Proceedings of the Royal Society of New South Wales 1942; 76: 203-224.

3. Archard JF. The temperature of rubbing surfaces. Wear 1959; 2: 438-455.

4. Barber JR. Distribution of heat between sliding surfaces. Journal of Mechanical Engineering Science 1967; 9: 351-354.

5. Vick B and Furey MJ. A basic theoretical study of the temperature rise in sliding contact with multiple contacts. Tribology International 2001; 34: 823-829. 
6. Salti B and Laraqi N. 3-D numerical modelling of heat transfer between two sliding bodies: temperature and thermal contact resistance. International Journal of Heat and Mass Transfer 1999; 42: 2363-2374.

7. Gao J, Lee SC, Ai X et al. An FFT-based transient flash temperature model for general threedimensional rough surface contacts. Trans. ASME Journal of Tribology 2000; 122: 519-523.

8. Olver AV. Testing transmission lubricants: the importance of thermal response. Proceedings of the IMechE - Part G: Journal of Aerospace Engineering 1991; 205: 35-44.

9. Merritt HE. Gear tooth contact phenomena. Proceedings of the IMechE 1962; 176: 141-163.

10. Clarke A, Sharif KJ Evans HP et al. Heat partition in rolling/sliding elastohydrodynamic contacts. Trans. ASME Journal of Tribology 2006; 128: 67-78.

11. Patching MJ, Kweh CC, Evans HP et al. Conditions for scuffing failure of ground and superfinished steel disks at high sliding speeds using a gas-turbine engine oil. Trans. ASME Journal of Tribology 1995; 117: 482-489.

12 Clarke A, Sharif KJ, Evans HP et al. Elastohydrodynamic modelling of heat partition in rollingsliding contacts. Proceedings of the IMechE - Part J: Journal of Engineering Tribology 2007; 221: 223-235.

13 Anderson $\mathrm{T}$ and Saunders OA. Convection from an isolated heated horizontal cylinder rotating about its axis. Proc. Roy. Soc. Lond. A. 1953; 217: 555-562.

14 Dropken D and Carmi A. Natural-Convection Heat Transfer From a Horizontal Cylinder Rotating in Air. Trans. ASME 1957; 79: 741-749.

15 Cardone G, Astarita T and Carlomango GM. Heat Transfer Measurement on a Rotating Disk. International Journal of Rotating Machinary 1997; 3: 1-9.

16 Özerdem B. Measurement of convective heat transfer coefficient for a horizontal cylinder rotating in quiescent air. International Communications in Heat and Mass Transfer 2000; 27: 389-395. 
17 Snidle RW, Dhulipalla AK, Evans HP and Cooper CV. Scuffing performance of a hard coating under EHL conditions at sliding speeds up to $16 \mathrm{~m} / \mathrm{s}$ and contact pressures up to $2.0 \mathrm{GPa}$. Trans ASME Journal of Tribology 2008; 130(2): 1-10. 